\title{
The transcriptomic analysis of Scots pine trees from the Chernobyl zone reveals pattern of adaptation to chronic radiation exposure
}

\author{
Volkova P.Yu. ${ }^{1 *}$, Duarte G.T. ${ }^{2}$, Geras'kin S.A. ${ }^{1}$ \\ ${ }^{1}$ Russian Institute of Radiology and Agroecology, Obninsk, Russia \\ ${ }^{2}$ Institut Jean-Pierre Bourgin, Versailles, France \\ * e-mail:volkova.obninsk@gmail.com
}

Radioactive contamination of the natural areas is one of the most long-lasting anthropogenic impacts on the environment. Scots pine (Pinus sylvestris L.) is an important species for radiation protection of biota due to to its high radiosensitivity, although the genome size of Pinacea species has imposed obstacles for high-throughput radiation-related studies so far. We conducted the analysis of the de novo assembled transcriptome of Scots pine populations growing in the Chernobyl-affected zone, which is still today contaminated with radionuclides. The transcriptional response indicates a continuous modulation of the cellular redox system, enhanced expression of chaperones and histones, along with the control of ions balance. Our data suggest that the modulation of ROS level occurs mainly through the control of glutathione- and thioredoxin-related responses and most likely involves a fine-tuning of ROS-generating processes. The SNPs analysis indicated enrichment of polymorphisms occurring on transcripts related to the antioxidant system and oxidation-reduction processes. While ROS-generating processes are being repressed, several pieces of evidence suggest the occurrence of control of gas exchanges via stomata, what would directly impact on the photosynthesis rate, a major ROS source. It is interesting to note that the control of stomata movement, although relying on several ABA target genes, apparently does not involve the modulation of ABA levels. A perspective for future work is the confirmation of ABA attenuation and of the control of photosynthesis rate on chronically irradiated plants. These adaptive responses are triggered by radiation doses 30 times lower than the one accepted as a safe for biota species by international regulations. This suggests that the environmental management in radiation protection should be reviewed. 